\title{
Shape interior modeling and mass property optimization using ray-reps
}

\author{
Wu, Jun; Kramer, Lou; Westermann, Rüdiger
}

Published in:

Computers \& Graphics

Link to article, DOI:

10.1016/j.cag.2016.05.003

Publication date:

2016

Document Version

Peer reviewed version

Link back to DTU Orbit

Citation (APA):

Wu, J., Kramer, L., \& Westermann, R. (2016). Shape interior modeling and mass property optimization using ray-reps. Computers \& Graphics, 58, 66-72. https://doi.org/10.1016/j.cag.2016.05.003

\section{General rights}

Copyright and moral rights for the publications made accessible in the public portal are retained by the authors and/or other copyright owners and it is a condition of accessing publications that users recognise and abide by the legal requirements associated with these rights.

- Users may download and print one copy of any publication from the public portal for the purpose of private study or research.

- You may not further distribute the material or use it for any profit-making activity or commercial gain

- You may freely distribute the URL identifying the publication in the public portal

If you believe that this document breaches copyright please contact us providing details, and we will remove access to the work immediately and investigate your claim. 


\title{
Shape Interior Modeling and Mass Property Optimization Using Ray-reps
}

\author{
Jun Wu*1, Lou Kramer ${ }^{2}$, Rüdiger Westermann ${ }^{2}$ \\ ${ }^{1}$ Department of Mechanical Engineering, Technical University of Denmark, Lyngby, Denmark \\ ${ }^{2}$ Department of Informatics, Technische Universität München, Garching, Germany \\ *Corresponding Author. Tel: +454525 4255. Fax: +454525 1961. Email: junwu@mek.dtu.dk
}

\begin{abstract}
We present a novel method for the modeling and optimization of the material distribution inside 3D shapes, such that their 3D printed replicas satisfy prescribed constraints regarding mass properties. In particular, we introduce an extension of ray-representation to shape interior modeling, and prove this parametrization covers the optimal interior regarding static and rotational stability criteria. This compact formulation thoroughly reduces the number of design variables compared to the general volumetric element-wise formulation. We demonstrate the effectiveness of our reduced formulation for optimizing shapes that stably float in liquids or spin around a prescribed axis.
\end{abstract}

Keywords: Mass properties, ray-reps, shape optimization, 3D printing

\section{Introduction}

Given the boundary surface representing the exterior of a 3D shape, we are interested in computing a material distribution in its interior, such that the resultant mass properties (i.e., the center of mass and the moment of inertia) satisfy a set of stability criteria. This design problem becomes very relevant in the era of 3D printing for fabricating customized shapes which stand [1, 2], spin [3], or float $[4,5]$ in a prescribed orientation.

A general formulation to this design problem is shape optimization on a volumetric basis $[1,5]$. To this end, the shape interior is discretized by hexahedral elements known as voxels, and each voxel is assigned a design variable. This parametrization results in a large number of design variables for accurately representing complex shapes.

To reduce the number of design variables, Bächer et al. [3] employ an adaptive octree grid where the cells split or merge on the fly during the optimization process. Musialski et al. [4] propose shape optimization by treating the thickness of the surface shell as design variables. Together with a reduced parametrization of offset surfaces, this formulation was demonstrated for static and rotational stability. However, the shell representation restricts the solution space, and thus an optimal solution that lies outside of this space cannot be reached.

In this paper, we present a reduced yet complete parametrization of shape interior, specifically designed for optimizing mass properties. Based on an analysis of mass properties and stability criteria, we prove that the optimal solution lies in a subspace represented compactly by an extension of ray-representations (ray-reps), which represent the shape by its intersections with parallel rays. In particular, we extend the concept of ray-reps by enhancing each ray with intervals distinguishing solid and void phases along this ray. The exact values of these intervals are automatically determined by an optimizer for controlling mass properties.

The specific contributions of our paper include:

- A compact parametrization of shape interior, which significantly reduces the number of design variables in shape interior optimization, and

- Insights into the optimal solution of mass properties under static and rotational stability criteria, which prove completeness of the reduced formulation, i.e., the optimal solution lies in the reduced space.

\section{Ray-reps for Shape Interior Modeling}

We start by briefly introducing ray-reps for solid modeling, and then go on to the extended version for modeling the shape interior which can be partially void.

Ray-reps for Solids. Ray-rep is a compact boundary representation, and it was introduced to the solid modeling community by Ellis et al. [6]. Similar approaches using the intersections between the surface mesh and parallel rays have been proposed, e.g., marching intersections [7], layered depth-normal images [8, 9]. Ray-rep is based on the parametrization of an object's surface using a set of parallel rays starting at a 2D grid. The solid is then represented by the sequence of intersections between its boundary surface and the rays. We assume rays start from a uniform grid on the $x y$-plane, and pierce along the $z$-axis which is aligned with the intended upright direction of the solid. As illustrated in Fig. 1 (left) on a 2D R-shaped solid, each ray has an even number of intersections with the watertight boundary surface. For each pair of intersections, we denote $z_{\min }$ to indicate the ray enters the solid, and $z_{\max }$ the ray exits the solid. 

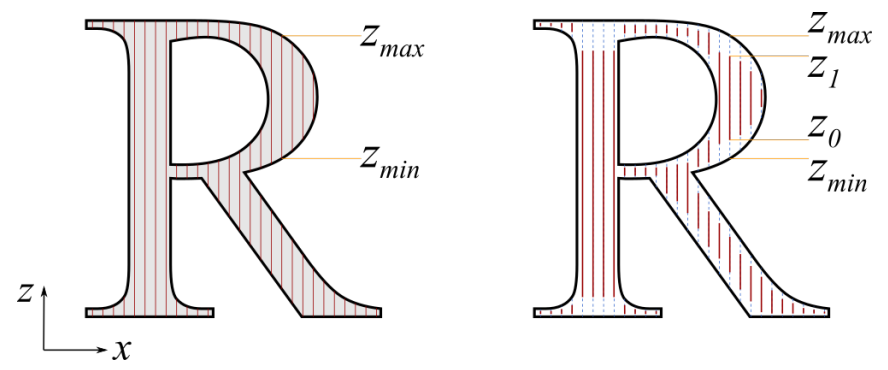

Figure 1: Left: A solid of letter ' $\mathrm{R}$ ' is represented by the intersections between its boundary (black) and the rays (red), i.e., $z_{\min }$ and $z_{\max }$ for each pair of intersections. Right: Two design variables $z_{0}$ and $z_{1}$ between each pair of intersections are introduced for modeling shape interior which is not necessarily fully solid.

We then collect all pairs of intersections by

$$
\operatorname{Ray}_{\text {solid }}^{i}=\left\{x^{i}, y^{i}, z_{\min }^{i}, z_{\max }^{i}\right\}
$$

where $x^{i}$ and $y^{i}$ are the $x y$-coordinates of the ray origin.

Rap-reps for Shape Interior. To extend ray-reps to shape interior modeling, we need intervals distinguishing solid and void regions along each ray. As will be proven in the next section, between each pair of intersections, there exists at most one solid region in the optimal solution under static and rotational stability criteria. Therefore we introduce two design variables $z_{0}^{i}$ and $z_{1}^{i}$, with $z_{\text {min }}^{i} \leq z_{0}^{i} \leq z_{1}^{i} \leq z_{\max }^{i}$, to represent the inner solid region. The ray-rep for shape interior is

$$
\operatorname{Ray}_{\text {interior }}^{i}=\left\{x^{i}, y^{i}, z_{\min }^{i}, z_{\max }^{i}, z_{0}^{i}, z_{1}^{i}\right\}
$$

Here $\overline{z_{0}^{i} z_{1}^{i}}$ is solid while both $\overline{z_{\text {min }}^{i} z_{0}^{i}}$ and $\overline{z_{1}^{i} z_{\max }^{i}}$ are void. In the extreme case of $z_{0}^{i}=z_{1}^{i}$, this ray is fully void; while in the extreme case of $z_{\min }^{i}=z_{0}^{i}$ and $z_{1}^{i}=z_{\max }^{i}$, the ray is fully solid. The values of $z_{0}^{i}$ and $z_{1}^{i}$ are to be determined by the optimizer. We note that a similar extension of ray-reps for modeling heterogeneous materials has been presented by Wang [10].

This representation inherits the compactness and simplicity of original ray-reps, and thus provides a reduced parametrization for shape optimization as we will explore.

\section{Optimizing Mass Properties on Ray-reps}

\subsection{Mass properties}

The stability of an object is determined by its mass properties, which include the total mass $m$, the center of mass $c=$ $\left(c_{x}, c_{y}, c_{z}\right)^{T}$, and the $3 \times 3$ symmetric moment of inertia $I=$ $\left(\begin{array}{lllllllll}I_{x x} & I_{x y} & I_{x z} ; I_{y x} & I_{y y} & I_{y z} ; I_{z x} & I_{z y} & I_{z z}\end{array}\right)^{T}$. The mass properties are given by volume integrals over the domain of the object. With the ray-reps for shape interior, and assuming a constant material density $\rho$ for the solid parts, the volume integrals are expressed as summation of line integrals over all rays:

$$
\begin{aligned}
m & =\rho A \sum_{i} \int_{z_{0}^{i}}^{z_{1}^{i}} d z, \\
c_{t} & =\frac{\rho A}{m} \sum_{i} \int_{z_{0}^{i}}^{z_{1}^{i}} t d z, t \in\{x, y, z\}, \\
I_{t t} & =\rho A \sum_{i} \int_{z_{0}^{i}}^{z_{1}^{i}}\left(u^{2}+v^{2}\right) d z,\{t, u, v\}=\{x, y, z\}, \\
I_{u v} & =I_{v u}=-\rho A \sum_{i} \int_{z_{0}^{i}}^{z_{1}^{i}} u v d z,\{u, v\} \subset\{x, y, z\},
\end{aligned}
$$

where $A$ is the area represented by a ray in the $x y$-plane. $A$ is constant since the rays are sampled uniformly, and its value depends on the sampling resolution.

\subsection{Static stability}

The static status of standing and floating of an object is related to its center of mass. Here we explain our formulation and prove its completeness on floating, while an extension to standing can be easily derived accordingly.

The object floats if its buoyancy balances the gravity, and its center of mass should be at its lowest possible position, in order to maximize floating stability. For simplicity, we choose a coordinate frame such that the $z$-axis coincides with the intended upright direction. The design problem is formulated

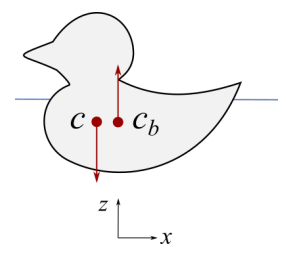
as

$$
\begin{array}{ll}
\underset{z_{0}, z_{1}}{\operatorname{minimize}} & c_{z}, \\
\text { subject to } & \left(c_{x}, c_{y}\right)=\left(c_{b, x}, c_{b, y}\right), \\
& m=m_{b}, \\
& z_{\text {min }}^{i} \leq z_{0}^{i} \leq z_{1}^{i} \leq z_{\text {max }}^{i}, \forall i,
\end{array}
$$

where $\left(c_{b, x}, c_{b, y}\right)$ is the $x y$-coordinates of the buoyant center, i.e., the centroid of the displaced volume of fluid, and $m_{b}$ is the mass of the displaced fluid. We postpone the calculation of $c_{b}, m_{b}$, and the verification of floating stability to Sec. 3.4.

This reduced formulation is said to be complete in the sense that the optimal solution in the general volumetric element-wise formulation lies within the space defined by the above constraints on ray-reps.

Proof of Completeness. Suppose in the volumetric elementwise formulation, the optimal solution contains two separated solid segments between a pair of intersections, denoted by $\overline{z_{a}^{i} z_{b}^{i}}$ and $\overline{z_{c}^{i} z_{d}^{i}}$, with the order $z_{\text {min }}^{i} \leq z_{a}^{i}<z_{b}^{i}<z_{c}^{i}<z_{d}^{i} \leq z_{\max }^{i}$, see Fig. 2 (left). We can easily find a replacement of these two solid segments by one solid segment $\overline{z_{0}^{i} z_{1}^{i}}$, with $z_{0}^{i}=z_{a}^{i}$, and $z_{1}^{i}=z_{0}^{i}+z_{b}^{i}-z_{a}^{i}+z_{d}^{i}-z_{c}^{i}$, see Fig. 2 (right). This replacement does not alter the constraint Eq. 4 -5, i.e., $c_{x}, c_{y}$, nor $m$, but reduces the objective Eq. 3 , i.e., $c_{z}$. This basically means that the optimal solution should contain at most one solid segment 

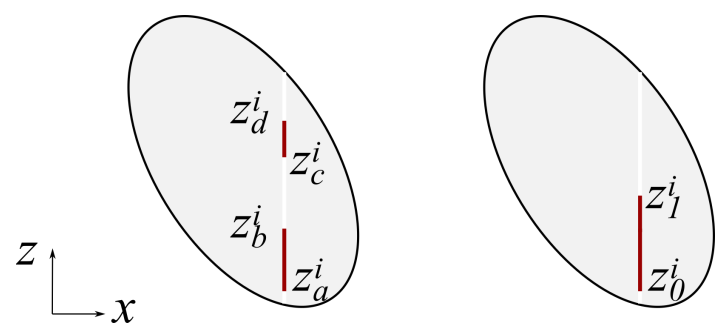

Figure 2: Illustration of material distribution along a ray in a 2D ellipse object. Replacing the solid segments $\overline{z_{a}^{i} z_{b}^{i}}$ and $\overline{z_{c}^{i} z_{d}^{i}}$ (left) by a single solid segment $\overline{z_{0}^{i} z_{1}^{i}}$ (right), with $z_{0}^{i}=z_{a}^{i}$, and $z_{1}^{i}=z_{0}^{i}+z_{b}^{i}-z_{a}^{i}+z_{d}^{i}-z_{c}^{i}$, does not move the center of mass along the $x$-axis, but brings it down along the $z$-axis.

along each ray, since otherwise it can be further optimized by this replacement.

Actually, in static stability, this formulation can be further simplified by $z_{0}^{i}=z_{\min }^{i}$, i.e., the solid segment, if exists, starts always from the lowest position. This is in line with the objective to minimize $c_{z}$. This fact is employed to further reduce the number of design variables by one half.

\subsection{Rotational stability}

Stable spinning around an axis is achieved if the axis is the smallest or largest principle axis of inertia. Additionally, the center of mass should also be kept lower to maintain a better stability. We align the spinning axis with the $z$-axis of the coordinate frame. Following the literature [3, 4], we formu-

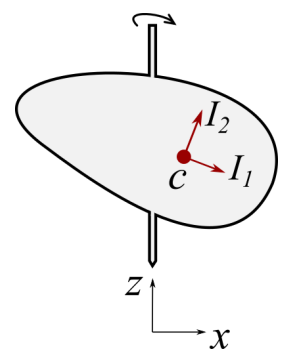
late the design problem as

$$
\begin{array}{cl}
\underset{z_{0}, z_{1}}{\operatorname{minimize}} & f=\lambda c_{z}+\frac{1}{I_{z z}^{2}}\left(\tilde{I}_{x x}^{2}+\tilde{I}_{y y}^{2}+2 I_{x y}^{2}\right), \\
\text { subject to } & \left(c_{x}, c_{y}\right)=(0,0), \\
& \left(I_{x z}, I_{y z}\right)=(0,0), \\
& z_{\min }^{i} \leq z_{0}^{i} \leq z_{1}^{i} \leq z_{\max }^{i}, \forall i,
\end{array}
$$

where $\lambda$ is a weighting factor, $\tilde{I}_{t t}=I_{t t}-\frac{s_{z}^{2}}{m}, t \in\{x, y\}$, with $s_{z}=\rho A \sum_{i} \int_{z_{0}^{i}}^{z_{1}^{i}} z d z$. The correction term $-\frac{s_{z}^{2}}{m}$ is introduced by applying the parallel axis theorem, since the origin of the chosen coordinate frame is in general not the same as the resultant center of mass [3].

Proof of Completeness. In a similar manner to the proof in static stability, we suppose in the volumetric element-wise formulation, the optimal solution contains two separated solid segments in the same ray, denoted by $\overline{z_{a}^{i} z_{b}^{i}}$ and $\overline{z_{c}^{i} z_{d}^{i}}$, with $z_{a}^{i}<z_{b}^{i}<$ $z_{c}^{i}<z_{d}^{i}$. Enforcing the replacement $\overline{z_{0}^{i} z_{1}^{i}}$ does not change $c_{x}$ and $c_{y}$ whose integrands have a degree of 0 wrt. $z$, nor $I_{x z}$ and $I_{y z}$ whose integrands have a degree of 1 , i.e.,

$$
\begin{gathered}
\int_{z_{0}^{i}}^{z_{1}^{i}} d z=\int_{z_{a}^{i}}^{z_{b}^{i}} d z+\int_{z_{c}^{i}}^{z_{d}^{i}} d z \\
\int_{z_{0}^{i}}^{z_{1}^{i}} z d z=\int_{z_{a}^{i}}^{z_{b}^{i}} z d z+\int_{z_{c}^{i}}^{z_{d}^{i}} z d z
\end{gathered}
$$

we arrive at

$$
z_{0}^{i}=\frac{1}{2}\left(\frac{\beta}{\alpha}-\alpha\right) \text { and } z_{1}^{i}=\frac{1}{2}\left(\frac{\beta}{\alpha}+\alpha\right),
$$

with $\alpha=z_{b}^{i}-z_{a}^{i}+z_{d}^{i}-z_{c}^{i}$ and $\beta=\left(z_{b}^{i}\right)^{2}-\left(z_{a}^{i}\right)^{2}+\left(z_{d}^{i}\right)^{2}-\left(z_{c}^{i}\right)^{2}$.

It can be proven that the replacement $\overline{z_{0}^{i} z_{1}^{i}}$ indeed reduces the objective function $f$. In fact, since the integrands of degree 0 and 1 vanish, the components $c_{z}, m, s_{z}, I_{x y}$ and $I_{z z}$ in the object function remain unchanged. Thus the comparison is concerned only with the $I_{x x}$ and $I_{y y}$ whose integrands have a degree of 2 , leading to

$$
\begin{aligned}
& f^{i}\left(z_{a}^{i}, z_{b}^{i}, z_{c}^{i}, z_{d}^{i}\right)-f^{i}\left(z_{0}^{i}, z_{1}^{i}\right) \\
= & \frac{2 \rho A}{I_{z z}}\left(\int_{z_{a}^{i}}^{z_{b}^{i}} z^{2} d z+\int_{z_{c}^{i}}^{z^{i}} z^{2} d z-\int_{z_{0}^{i}}^{z_{1}^{i}} z^{2} d z\right) \\
= & \frac{2 \rho A\left(z_{a}^{i}-z_{b}^{i}\right)\left(z_{b}^{i}-z_{c}^{i}\right)\left(z_{c}^{i}-z_{d}^{i}\right)\left(z_{d}^{i}-z_{a}^{i}\right)}{I_{z z}\left(z_{a}^{i}-z_{b}^{i}+z_{c}^{i}-z_{d}^{i}\right)} .
\end{aligned}
$$

Since $z_{a}^{i}<z_{b}^{i}<z_{c}^{i}<z_{d}^{i}$, the above form is always positive. This confirms that the replacement of two separated solid segments by one solid segment leads to a more optimal solution. Therefore, the optimal solution for rotational stability also contains at most one solid segment in each ray, and thus lies within the space defined on the extended ray-reps.

\subsection{Implementation}

The input to our algorithm is the exterior boundary surface which is conventionally represented by a triangle mesh $\mathcal{M}$. The user prescribes the intended orientation of the model, the density of solid material, for floating the intended submerged height and the density of fluid, and for spinning the intended spinning axis. The output is a mesh composited of two parts, the input surface mesh and the boundary surface of the carved interior.

Discretization. To retain the given surface mesh, we apply rayreps to a sub-domain which is completely inside the mesh. The surface mesh is first discretized into a uniform hexahedral model with a user-defined resolution in a conservative voxelization process, keeping voxels the centroid of which is inside the mesh. We then build a distance field and prescribe voxels whose distance value is smaller than a thickness value as boundary voxels. The sub-domain composed of remaining voxels is converted to ray-reps as the design domain. The contribution of the boundary layer to the mass properties is calculated by doing surface integrals, and added to the overall mass properties. After the optimization process, the continuous design variables are rounded to the closest voxel height, and then the interior surface is constructed from the faces between solid and void voxels.

Buoyancy Properties. Besides the center of buoyancy $c_{b}$ and the mass of displaced liquid $m_{b}$, the metacentre $c_{m}$ is an important attribute determining the stability of floating. As illustrated in Fig. 3, metacentre $c_{m}$ is the intersection point between an imaginary vertical line passing through the center of buoyancy 


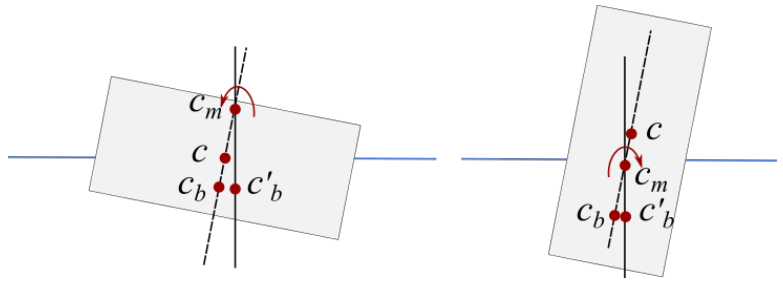

Figure 3: Illustration of stable (left) and unstable (right) floating configuration of a rectangular shape. On the left, the center of mass $c$ is located below the metacentre $c_{m}$, and thus creates a correction torque around $c_{m}$ to restore stability, while on the right, the torque aggravates the turn-over.

$c_{b}$ and the imaginary vertical line through a new center of buoyancy $c_{b}^{\prime}$ when the object is slightly tipped [11]. If the center of mass is below the metacentre (i.e., $c_{z}<c_{m, z}$ ), gravity restores stability when the object is tipped (cf. Fig. 3 left). Otherwise gravity tends to aggravate the turn-over (cf. Fig. 3 right).

Since the outer surface mesh remain unchanged, the buoyancy properties are calculated directly on the triangle mesh. In particular, we first find the intersection between the surface mesh and the waterplane. Then the properties are calculated by using the surface integrals over the submerged surface and the waterplane.

$$
\begin{aligned}
& m_{b}=\rho_{w} V_{b} \\
& c_{m}=c_{b}+\left(0,0, \frac{I_{w}}{V_{b}}\right)
\end{aligned}
$$

where $V_{b}$ is the volume of displaced liquid, $\rho_{w}$ the density of liquid, $I_{w}$ the smallest second moment of the intersection plane. Note that these calculations are independent of the interior of the shape, since the void interior also effectively displaces liquid.

Numerical Optimization. We solve the optimization problems (Eq. 3-6 and Eq. 7-10) by using the interior point method. In particular, we employ the open source package Ipopt [12], which handles nonlinear, nonconvex, but twice continuously differentiable functions. It iteratively searches for a (local) optimal solution by evaluating the objective function, the constraint functions, and the derivatives of these functions with respect to the design variables (i.e., $z_{0}^{i}$ and $z_{1}^{i}$ ). Since these functions are composed of polynomials, derivatives can be derived analytically, e.g., $\frac{\partial m}{\partial z_{1}^{i}}=\rho A$ and $\frac{\partial m}{\partial z_{0}^{i}}=-\rho A$.

Due to the large number of rays, many constraints of the form $z_{\min }^{i} \leq z_{0}^{i} \leq z_{1}^{i} \leq z_{\max }^{i}$ have to be considered in the optimization process. On the other hand, from the analysis of static stability it is known that solid parts start from the bottom. Thus, the per-ray constraints are physically equivalent to fixed lower and upper bounds on $z_{1}^{i}$ as

$$
z_{\min }^{i} \leq z_{1}^{i} \leq z_{\max }^{i}
$$

with enforced $z_{0}^{i}=z_{\min }^{i}$. As opposed to general constraints, lower and upper bounds on design variables can be handled very efficiently by optimization algorithms, so that the optimization process can be accelerated considerably. Furthermore,
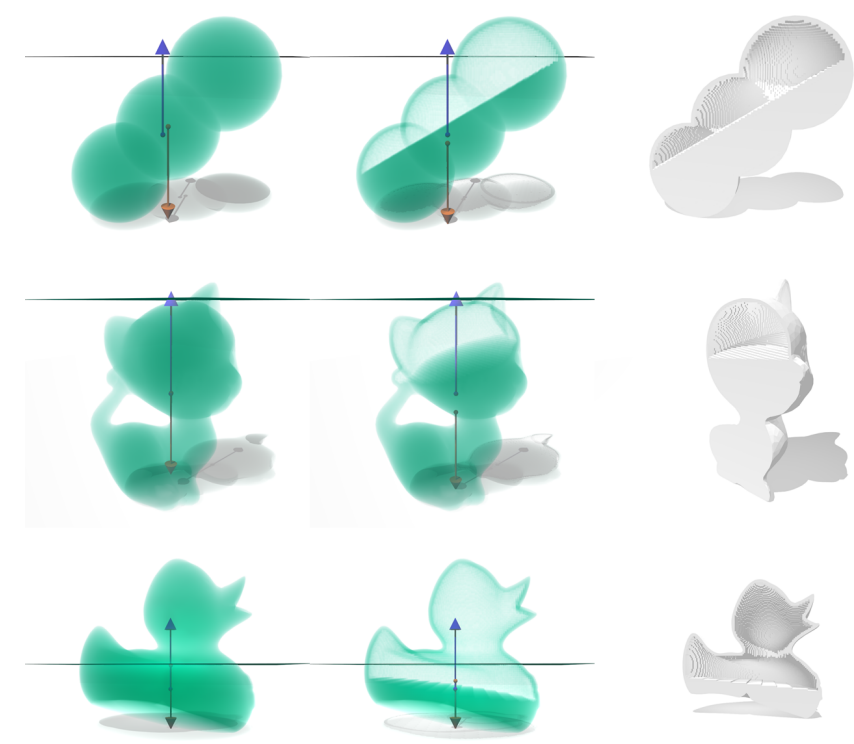

Figure 4: Shapes optimized for stable floating. On the left of each group, the fully solid shape does not satisfy the floating stability criteria. The middle one shows the optimized shape where the center of mass and the center of buoyancy are vertically aligned, and the center of mass is located in its lowest possible position. The right one reveals the interior cavity. The waterplane is indicated by a horizontal line in the left and middle images.

with this reduction, the static stability problem becomes convex: The objective in Eq. 3 is a convex quadratic function

$$
c_{z}=\frac{\rho A}{2 m} \sum_{i}\left(\left(z_{1}^{i}\right)^{2}-\left(z_{\min }^{i}\right)^{2}\right)
$$

and the constraints in Eq. 4 and 5 are linear functions of $z_{1}^{i}$.

For rotational stability, we convert the per-ray constraints to the following lower and upper bounds,

$$
\begin{aligned}
& z_{\min }^{i} \leq z_{0}^{i} \leq \frac{1}{2}\left(z_{\min }^{i}+z_{\max }^{i}\right), \\
& \frac{1}{2}\left(z_{\min }^{i}+z_{\max }^{i}\right) \leq z_{1}^{i} \leq z_{\max }^{i} .
\end{aligned}
$$

This approximation implicitly enforces the condition $z_{0}^{i} \leq z_{1}^{i}$. Due to the complexity of the rotational stability problem, the optimized solution is not guaranteed to be globally optimal. However, we have performed various optimizations starting from different initial values (e.g., $z_{0}$ and $z_{1}$ start from their lower bounds, upper bounds, or middle points), all of them showing the objective converges to the same minimum.

\section{Results}

The proposed formulation has been implemented and tested on a number of floating and spinning shapes.

Examples. Our first example is a set of models optimized for floating in water, as shown in Fig. 4. After optimization, the 

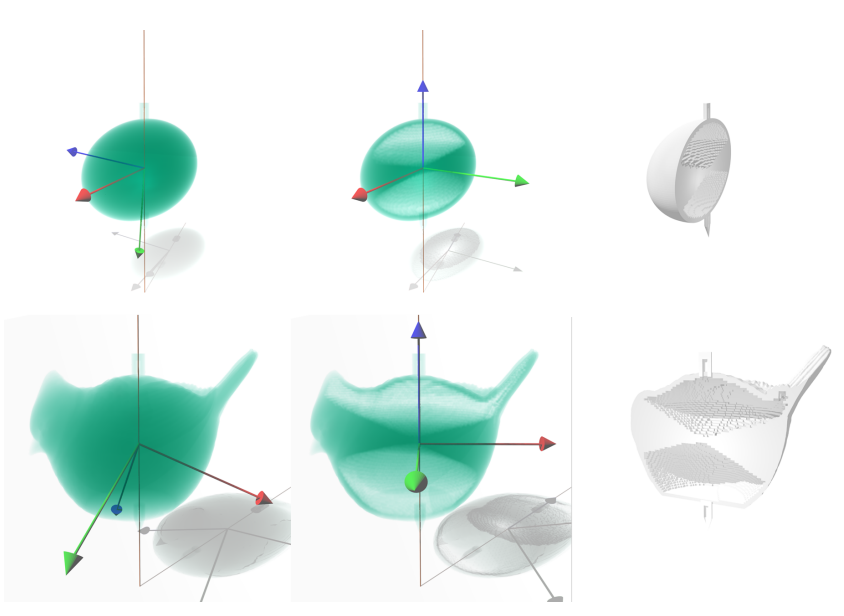

Figure 5: Shapes optimized for stable spinning. On the left of each group, the fully solid shape does not satisfy the spinning stability criteria. The middle one shows the optimized version where the principle axis is aligned with the predefined spinning axis. The right version reveals the interior cavity.

center of mass is aligned vertically with the center of buoyancy, and the magnitude of the buoyant force and the magnitude of the gravity force are the same. Not surprisingly, the cavity is located in the top of each model. This agrees with the objective to bring down the center of mass.

Two spinning models are shown in Fig. 5. After optimization, one of the principal axes of inertia is aligned with the prescribed spinning axis. On the vertical cross-section as shown on the right, it can be observed that the optimized spinning models have a ' $\vee$ '-shaped cavity to the top and a ' $\wedge$ '-shaped cavity to the bottom. It is intuitive that on a horizontal cross-section, the material tends to spread far away from the center, in order to maximize the inertia around the spinning axis. In the vertical line, the material tends to be close (vertically) to the center of mass, and thus to reduce the inertia around the other two principle axes.

We take the bird model as an example to analyze the numerical results. The moment of inertia $\left(I_{x x}, I_{y y}, I_{z z}, I_{x y}, I_{y z}, I_{x z}\right)$ before and after optimization is

$$
(110.0,138.5,143.2,-10.7,-1.9,19.5)
$$

and

$$
(64.0,78.7,98.4,-7.9,0.0,0.0)
$$

respectively. It can be seen that after optimization $I_{y z}$ and $I_{x z}$ vanish, making the $z$-axis as one of the principle axes. Transforming the inertia tensor to principle values, we get $\left(I_{1}, I_{2}, I_{3}\right)=$ $(60.5,82.2,98.4)$, with $I_{3}=I_{z z}$. It satisfies the stability condition that the spinning $z$-axis is the largest principle axis of inertia.

Prints. We have fabricated some of the optimized models by $3 \mathrm{D}$ printing, with metallic plastic materials $\left(\rho=1.36 \mathrm{~g} / \mathrm{cm}^{3}\right)$. The models are cut into two pieces to reveal the cavities (see Fig. 6), and are glued together upon printed. The number of isolated cavities is rather small, since as analyzed in our formulation no more than two void segments exist in each ray.
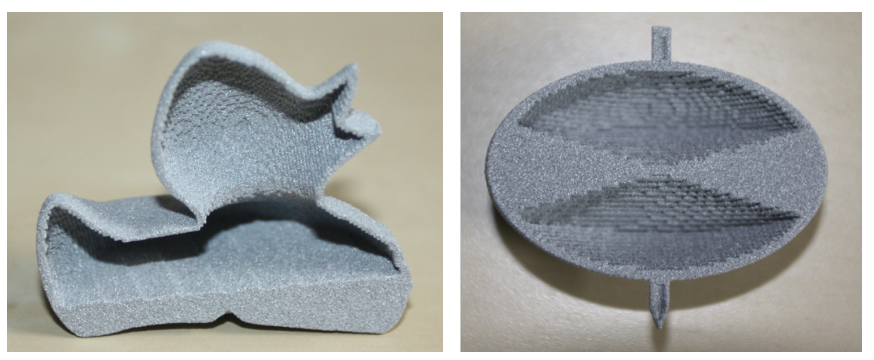

Figure 6: Prints showing the interior cavity.
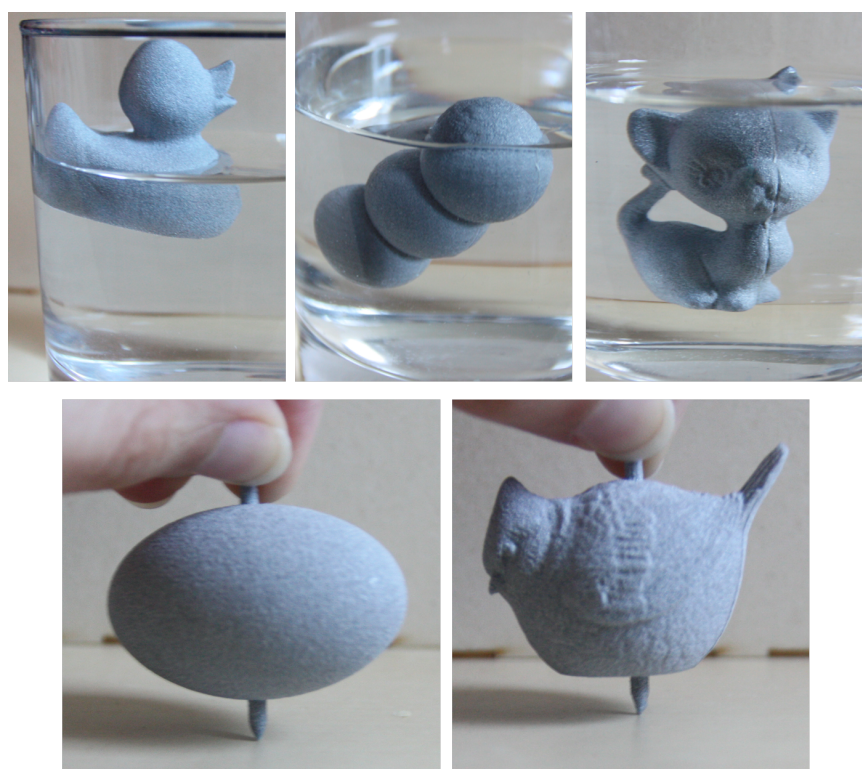

Figure 7: A collection of printed shapes in their designed function. The first row: Shapes which stably float in water. The second row: Shapes which can stably spin.

The fact that the number of cavities is small has been reported earlier in [3]. For models to be floating in water, since the material absorbs water and changes its density, we coat the shapes by waterproof spray. The 3D printed shapes in their designed function are collectively shown in Fig. 7.

Performance. The models shown in this paper are discretized on a volumetric resolution close to $100^{3}$. The number of resultant design variables using the ray-reps is between $4 \mathrm{k}$ to $9 \mathrm{k}$. The optimization converges within a few seconds using the optimization package Ipopt [12] with its default setting. The detailed model information and timings are given in Table 1. The tests are performed on a standard desktop PC with an Intel Xeon E5-1620 processor and 8GB of RAM.

Comparison. We compare our reduced formulation with the standard volumetric element-wise formulation for spinning optimization. Table 2 lists the timings for solving the reduced problem and the timings for solving the standard problem on different resolutions. We can observe a speed-up factor of 4 to 20 , varying on the resolution of the volumetric discretization. 


\begin{tabular}{crrr} 
Model & Resolution & \# Variables & Time $(\mathrm{s})$ \\
\hline 3-spheres & $51 \times 101 \times 101$ & 4236 & 0.499 \\
Kitten & $101 \times 92 \times 154$ & 8176 & 0.919 \\
Duck & $89 \times 105 \times 101$ & 8087 & 0.799 \\
Ellipsoid & $100 \times 65 \times 63$ & 8682 & 3.870 \\
Bird & $100 \times 75 \times 75$ & 8776 & 3.975
\end{tabular}

Table 1: Performance for shape interior optimization using ray-reps. The first three models are optimized for floating, while the last two are optimized for spinning.

\begin{tabular}{crrrrr} 
& \multicolumn{2}{c}{ Ray-reps } & \multicolumn{2}{c}{ Voxels } & Speed- \\
\cline { 2 - 5 } Resolution & \# Var. & T. $(s)$ & \# Var. & T. (s) & up \\
\hline $80 \times 52 \times 51$ & 5882 & 1.79 & 94975 & 34.74 & 19.41 \\
$64 \times 42 \times 41$ & 3706 & 0.71 & 46314 & 11.48 & 16.11 \\
$54 \times 35 \times 34$ & 2536 & 0.48 & 26783 & 3.48 & 7.25 \\
$40 \times 26 \times 26$ & 1390 & 0.31 & 10764 & 1.37 & 4.42 \\
$80 \times 60 \times 60$ & 6184 & 2.24 & 100224 & 17.94 & 8.00 \\
$54 \times 40 \times 40$ & 2646 & 0.72 & 28261 & 4.43 & 6.15 \\
$40 \times 30 \times 30$ & 1414 & 0.29 & 11306 & 2.20 & 7.59
\end{tabular}

Table 2: Performance comparison between optimization using ray-reps and voxels. The first four rows give timings for the ellipsoid model, while the last three refer to the bird model.

\section{Conclusion}

We have presented shape interior optimization based on extended ray-reps. Our formulation significantly reduces the number of design variables, at the same time it covers the optimal solution space regarding static and rotational stability criteria.

Our reduced formulation is designed for optimizing mass properties, and specifically for static and rotational stability problems. In a general volumetric optimization, e.g., structural optimization [13], the optimal shape is not contained in the space defined by the fixed number of rays. It would be interesting to duplicate rays during the optimization process in order to accommodate more complex topologies.

\section{Acknowledgement}

This work was partially supported by the H.C. Ørsted Postdoc Programme, which has received funding from the People Programme (Marie Curie Actions) of the European Union's Seventh Framework Programme (FP7/2007-2013) under REA grant agreement no. 609405 (COFUNDPostdocDTU), and partially supported by the European Union under the ERC Advanced Grant 291372 SaferVis - Uncertainty Visualization for Reliable Data Discovery.

\section{References}

[1] R. Prévost, E. Whiting, S. Lefebvre, O. Sorkine-Hornung, Make it stand: Balancing shapes for 3d fabrication, ACM Trans. Graph. 32 (4) (2013) 81:1-81:10. doi:10.1145/2461912.2461957.

URL http: //doi .acm.org/10.1145/2461912.2461957

[2] A. N. Christiansen, R. Schmidt, J. A. Brentzen, Automatic balancing of 3d models, Computer-Aided Design 58 (0) (2015) 236 - 241. doi:http://dx.doi.org/10.1016/j.cad.2014.07.009.
URL http://www.sciencedirect.com/science/article/pii/ S0010448514001614

[3] M. Bächer, E. Whiting, B. Bickel, O. Sorkine-Hornung, Spin-it: Optimizing moment of inertia for spinnable objects, ACM Trans. Graph. 33 (4) (2014) 96:1-96:10. doi : 10.1145/2601097.2601157.

URL http://doi .acm.org/10.1145/2601097.2601157

[4] P. Musialski, T. Auzinger, M. Birsak, M. Wimmer, L. Kobbelt, Reducedorder shape optimization using offset surfaces, ACM Trans. Graph. 34 (4) (2015) 102:1-102:9. doi : 10.1145/2766955.

URL http://doi.acm.org/10.1145/2766955

[5] L. Wang, E. Whiting, Buoyancy optimization for computational fabrication, Computer Graphics Forum (Proceedings of Eurographics) 35 (2).

[6] J. L. Ellis, G. Kedem, T. C. Lyerly, D. G. Thielman, R. J. Marisa, J. P. Menon, H. B. Voelcker, The ray casting engine and ray representatives, in: Proceedings of the First ACM Symposium on Solid Modeling Foundations and CAD/CAM Applications, SMA '91, ACM, New York, NY, USA, 1991, pp. 255-267. doi:10.1145/112515.112548. URL http://doi .acm.org/10.1145/112515.112548

[7] C. Rocchini, P. Cignoni, E. Ganovelli, C. Montani, P. Pingi, R. Scopigno, Marching intersections: an efficient resampling algorithm for surface management, in: Shape Modeling and Applications, SMI 2001 International Conference on., IEEE, 2001, pp. 296-305.

[8] C. C. Wang, Y.-S. Leung, Y. Chen, Solid modeling of polyhedral objects by layered depth-normal images on the $\{\mathrm{GPU}$, Computer-Aided Design 42 (6) (2010) 535 - 544 doi:http: //dx.doi.org/10.1016/j.cad.2010.02.001.

URL http://www.sciencedirect.com/science/article/pii/ S0010448510000278

[9] Y. S. Leung, C. C. L. Wang, Conservative sampling of solids in image space, IEEE Computer Graphics and Applications 33 (1) (2013) 32-43. doi : 10.1109/MCG.2013.2.

[10] C. C. L. Wang, Computing on rays: A parallel approach for surface mesh modeling from multi-material volumetric data, Comput. Ind. 62 (7) (2011) 660-671. doi:10.1016/j.compind.2011.02.004. URL http://dx.doi.org/10.1016/j. compind.2011.02.004

[11] B. Barrass, C. D. Derrett, Ship stability for masters and mates, Butterworth-Heinemann, 2011.

[12] A. Wächter, L. T. Biegler, On the implementation of an interior-point filter line-search algorithm for large-scale nonlinear programming, Math. Program. 106 (1) (2006) 25-57. doi : 10.1007/s10107-004-0559-y. URL http: //dx.doi.org/10.1007/s10107-004-0559-y

[13] J. Wu, C. Dick, R. Westermann, A system for high-resolution topology optimization, IEEE Transactions on Visualization and Computer Graphics 22 (3) (2016) 1195-1208. doi : 10.1109/TVCG . 2015 . 2502588. 